\title{
sur la gestion et les finances publiques En Septembre 2018
}

\section{FINANCES DE L'ÉTAT ET DES OPÉRATEURS}

\section{$\lambda$ La dette a atteint 100\% du PIB en 2017}

Par un communiqué du 6 septembre 2018, I'INSEE vient de réajuster les indicateurs de la dette publique et de déficit à la fin 2017. Alors que l'estimation précédente de la dette notifiée s'établissait à 96,8\% du PIB, celle-ci atteint $2257 \mathrm{Md} €$ soit $98,5 \%$ du PIB en raison de l'intégration des comptes de SNCF réseau (35,8 Md€ en 2016 et 39,4 Md€ en 2017) dans les administrations publiques (APU) à partir de 2016. Cette décision est due au fait que les recettes commerciales de l'entreprise publique ne couvrent depuis 2016 que la moitié de ses coûts de production. Le déficit de l'exercice 2017 passe de 2,6\% à 2,7\% du PIB pour tenir compte du besoin de financement de SNCF réseau (3,2 Md€ en 2016 et 2,2 Md€ en 2017) et des 2,5 Md€ de recapitalisation d'Orano (nouveau nom d'Areva) considérés comme une dépense publique de l'exercice. Quelques jours après ce communiqué, le 28 septembre 2018, I'INSEE a indiqué dans son tableau récapitulatif de l'année 2017, que la dette publique avait dépassé temporairement la barre symbolique des $100 \%$ du PIB à la fin du deuxième trimestre (fin juin 2017) avec le chiffre de 100,9\%.

\section{$\lambda$ Les résultats du loto du patrimoine}

Le tirage spécial du loto du vendredi 14 septembre 2018 prévu par la loi de finances rectificative pour 2017 a été un grand succès et a permis la vente de plus de 2,5 millions de tickets. Les sommes issues des prélèvements de l'État que la Française des jeux a tiré des jeux du patrimoine, soit 15 à $20 \mathrm{M} €$, escomptés sont budgétés pour être reversées à la Fondation du patrimoine.

\section{$\lambda$ Présentation du projet de loi de finances pour 2019}

Le projet de loi de finances de l'État pour 2019 a été présenté au conseil des ministres du $24 \mathrm{sep}$ tembre 2018. II est fondé sur des hypothèses de croissance de 1,7\% pour les années 2018 et 2019 et d'inflation de 1,6 et 1,3\% que le Haut conseil des finances publiques a estimé « plausible » dans son avis du 19 septembre. Le déficit nominal est pour la troisième année consécutive inférieur aux $3 \%$. II est prévu à $2,6 \%$ du PIB en 2018 et $2,8 \%$ en 2019 (- 3,7\% pour l'État et les administrations centrales; $+0,1 \%$ pour le secteur local et $+0,8 \%$ pour la sécurité sociale). Corrigé des mesures exceptionnelles (notamment la transformation du CICE), le déficit public sera limité à 1,9\% en 2019. Le déficit structurel s'élèvera à - 2\% en 2019 et l'effort d'ajustement structurel est limité à 0,3\%, ce qui reste éloigné de nos engagements européens comme l'a relevé le HCFP. La progression des dépenses publiques en volume est limitée à 0,6\% (contre 1,4\% en 2017 et $0 \%$ en 2018). La part des dépenses publiques dans le PIB diminue à $54,6 \%$ en 2018 et $54 \%$ en 2019. Les prélèvements obligatoires régressent également à 45\% en 2018 et $44,2 \%$ en 2019. Plus de $25 \mathrm{Md} €$ d'allègements sont annoncés dont 18,8 Md€ net pour les entreprises (transformation du CICE en allègements pérennes de charges sociales plus de $20 \mathrm{Md} €$ et poursuite de la baisse de l'impôt sur les sociétés de 33 à $31 \%$ - 2,4 Md€). Les ménages bénéficieront de $6 \mathrm{Md} €$ d'allègements : 3,8 Md€ au titre de la $2^{\mathrm{e}}$ tranche du dégrèvement de la taxe d'habitation; 4,1 Md€ au titre de la baisse des cotisations sociales. La dette publique, après le réajustement de 2017, est stable à un niveau élevé : 98,7\% en 2018 et $98,6 \%$ en 2019. Pour l'État, les recettes nettes atteignent 291,4 Md€, les dépenses nettes 390,8 Md€. Le solde du budget général de l'État s'établit à - 98,7 Md€ après - 67,7 Md€ en exécution 2017 et - 81,3Md€ en 2018). Les dépenses pilotables de l'État se réduisent de - 0,5\% en volume et augmentent de $0,8 \%$ en valeur. Les missions : " défense », "sécurité » « justice », « immigration, asile, intégration » voient leurs moyens augmentés, au contraire des missions " cohésion des territoires », « agriculture », « travail et emploi ». Les effectifs de l'État et de ses opérateurs seront réduits de 4.164 emplois (- 1600 emplois en 2018) 
dont 1947 au ministère de l'action et des comptes publics.

\section{$\lambda$ Querelle de chiffres sur l'amélioration du pouvoir d'achat}

Une note de l'OFCE, publiée le 20 septembre 2018, fournit une estimation de l'incidence directe des mesures fiscales et sociales décidées par le Gouvernement sur le pouvoir d'achat global des ménages en 2019, calculée sur une moyenne annuelle en tenant compte des dates d'entrée en vigueur des mesures. Alors que dans le projet de loi de finances, le Gouvernement annonce des mesures en faveur des ménages à $6 \mathrm{Md} €$, l'institut de recherche les estime à 3,5 Md€ (après $0,1 \mathrm{Md} €$ en 2018). Les allègements de prélèvements sur les ménages s'élèvent à 9,4 Md€ (cotisations sociales : 4,5 Md€, taxe d'habitation : 3,5 Md€; prélèvement forfaitaire unique : $0,6 \mathrm{Md} €$; désocialisation des heures supplémentaires : 0,7 Md€; atténuation hausse de CSG petites retraites: 0,3 Md€; autres mesures : + 0,2 Md€). Les augmentations de fiscalité écologique et sur le tabac pèseront de 3,5 Md€, chiffre plus élevé que les 2,3 Md€ officiels qui prennent en considération la baisse de consommation induite. Les revalorisations de prestations sociales sont pour les chercheurs plus que compensées par la désindexation partielle des retraites et des prestations familiales (- $3 \mathrm{Md} €$ ) que les documents budgétaires ne prennent pas en compte. Si on ajoute la hausse des cotisations d'assurances complémentaires (AGirc-Arrco) pour 1,8 Md€, il ne resterait que 1,7 Md€ d'amélioration directe de pouvoir d'achat.

\section{$\lambda$ Rapport d'activité de l'Agence France Trésor}

Le rapport d'activité de l'Agence France Trésor confirme les excellentes conditions de placement de la dette publique en 2017. Le programme a porté sur $185 \mathrm{Md} €$ de dette à moyen et long terme (MLT) nets de rachats. Le taux moyen des émissions reste très modéré $(0,65 \%$ contre $0,37 \%$ en 2016). La maturité moyenne des émissions s'allonge $(11,8$ ans contre 11,5$)$ et la durée moyenne de la dette également $(7,8$ ans contre 7,5$)$. En 2017, I'Agence a lancé une nouvelle obligation à terme (OAT) à 30 ans, une OAT verte de $7 \mathrm{Md} €$ et a engagé le rapprochement de ses équipes avec celles de la CADES qui gère la dette sociale.

\section{$\lambda$ Modernisation de la gestion budgétaire et financière : révision du décret GBCP}

La démarche Action publique 2022 comprend un volet de modernisation de la gestion budgétaire et financière de l'État et des opérateurs. La pre- mière étape est réalisée par la révision du décret relatif à la gestion budgétaire et financière du 7 novembre 2012 (GBCP) par un décret $n^{\circ} 2018-80$ du 24 septembre 2018 publié au JORF le 25 septembre, texte $n^{\circ} 20$. Le texte supprime la comptabilité d'analyse des coûts qui sera remplacée par une « vraie » comptabilité analytique. II simplifie les règles applicables aux organismes. II supprime le contrôle de régularité des actes de gestion du personnel par les contrôleurs budgétaires et ouvre la possibilité d'une modulation des contrôles en fonction des résultats du contrôle interne budgétaire et des contrôles antérieurs. II ouvre la possibilité d'une adaptation des contrôles du service fait et la possibilité d'expérimentations de dérogations à certaines règles $d u$ décret GBCP.

\section{FinANCES LOCALES}

\section{$\lambda$ Ajustements des contrats de maîtrise des dépenses locales}

Le 19 septembre 2018 devant la conférence des villes organisée par l'Assemblée des communautés de France, le Premier ministre a ouvert la porte à un réajustement de la procédure et des contenus des contrats de maîtrise des dépenses publiques. Pour sa part, I'ACF demande cinq principaux correctifs : neutralisation des dépenses imposées par l'État, exclusion du périmètre des dépenses de fonctionnement des subventions d'investissement versés à un syndicat ou à un budget annexe, recours à un tiers de confiance en cas de divergences entre le préfet et la collectivité, neutralisation des flux croisés et des participations reçues dans le calcul des recettes de fonctionnement servant de base au plafonnement de la reprise. L'ADCF avait émis le 13 septembre 2018 des demandes similaires avec en plus un souhait de voir prises en compte les dépenses nettes plutôt que les dépenses brutes et un réajustement de la norme en cas d'accélération de l'inflation prévisionnelle.

\section{$\lambda$ Le rapport du Conseil d'évaluation des normes}

Le Conseil national d'évaluation des normes applicables aux collectivités territoriales (CNEN), coprésidé par Alain Lambert et Jean-Claude Boulard, aujourd'hui décédé, a publié son rapport le 13 septembre 2018. I| préconise un net renforcement du dispositif. Le rapport préconise un rappel des principes de proportionnalité, d'adaptabilité et de subsidiarité par un décret en Conseil d'État, une évaluation de l'ensemble des normes tous les dix ans, une sanction plus efficace en cas 
d'insuffisance d'étude d'impact (obligation de recourir à une étude extérieure à l'administration centrale concernée et arbitrage du Conseil d'État), un droit de dérogation confié aux communes sans autorisation préfectorale, un allongement du mandat et des moyens renforcés pour le médiateur des normes, la création d'une mission de simplification chargée de formuler des propositions sectorielles, un renfort de l'obligation d'évaluation du stock de normes par les ministères, une clarification des responsabilités dans I'instruction et le suivi des propositions de simplifications, la création d'une surprime simplification ou d'un indicateur simplification dans l'évaluation des secrétaires généraux et directeurs d'administration centrale, un pilotage interministériel clarifié autour du CNEN.

\section{$\lambda$ Reprise de l'investissement plus rapide que prévu}

La note de conjoncture de la Banque Postale publiée le 19 septembre 2019 confirme le ralentissement des dépenses de fonctionnement (+ 0,8\% à 183,9 Md€ dont $+1 \%$ pour les dépenses de personnel) qui sont très inférieures à la norme de 1,2\%. Les recettes de fonctionnement, sans baisse générale de dotations de l'État pour la première fois depuis quatre ans, progressent de 1,3\%. La Banque annonce pour 2018 une augmentation prévisible des dépenses d'investissement de $7 \%$ à $54,2 \mathrm{Md} €$ comparable à celle de 2017 (6,8\%) et supérieure aux prévisions publiées en mai $(6,1 \%)$. Le cycle électoral communal, la mise en œuvre des projets des nouvelles intercommunalités élargies et l'amélioration de la situation financière des départements peuvent expliquer ce rebond de l'investissement local qui rejoint le niveau de 2004. L'amélioration de l'épargne brute (+ 2,8\%, 42,8 Md€) s'accompagne d'une augmentation du recours à l'emprunt en 2018 (+ 4,5\% soit 17,3 Md€) mais le ratio d'encours de dettes, qui progresse de $0,5 \%$, est à son plus bas niveau depuis 2012 (7,8\% du PIB).

\section{$\lambda$ La place de la solidarité dans les budgets locaux}

La Banque Postale a procédé, dans sa dernière note de conjoncture, à une étude de la place de la solidarité dans les budgets locaux. La solidarité entre collectivités prend la forme des dispositifs de péréquation horizontale $(2,8 \mathrm{Md} €$, doublement depuis 2013) et verticale (6 Md€ mais de plus en plus « horizontalisés ») et de mécanismes locaux qui sont en baisse (subventions : 4,8 Md€ en forte baisse, dotation de solidarité communautaire en baisse et mutualisation en forte hausse). La solidarité des territoires à l'égard des citoyens prend majoritairement la forme des allocations individuelles, dépenses obligatoires de $30 \mathrm{Md} €$ principalement à la charge des départements et dont le poids a augmenté de 12\% entre 2013 et 2017, et de dépenses facultatives en net repli (insertion pour les départements, CCAS, subventions aux associations, sports, loisirs, culture).

\section{$\lambda$ Les finances des communes rurales}

Une étude de la Banque Postale, publiée le 24 septembre 2018, présente une analyse des finances des communes de moins de 3.500 habitants. Elles regroupent $33 \%$ de la population, gèrent $22 \mathrm{Md} €$ de dépenses, soit $24,8 \%$ des dépenses des autres communes et leur encours de dette s'élève à 13,6 Md€ soit $634 €$ par habitant (contre $1133 €$ pour les autres communes). Les situations sont évidemment très disparates selon la taille des communes qui sont classées par strates de population.

\section{त Rapport de la Cour des comptes sur les finances publiques locales}

La Cour des comptes a publié le 25 septembre 2018 son rapport sur la situation financière et la gestion des collectivités territoriales et de leurs établissements publics. La Cour constate que la baisse des concours financier de l'État entre 2013 et 2017 (de 58,2 Md€ à 47,1 Md€, soit - 19\%) a mis un "coup d'arrêt » à la croissance des dépenses : ralentissement des dépenses de fonctionnement ( $+1,5 \%$ en moyenne) et baisse de l'investissement (-11\%), même si l'effet de la réduction de la DGF a été atténuée par le dynamisme «naturel » de la fiscalité locale. Le rythme de croissance de la dette a lui aussi été ralenti et la capacité de désendettement à la fin de 2017 est correcte (bloc communal : 5,6 ans; départements; 4,2; régions : 4,9). Bien que l'année 2017 marque une nouvelle augmentation des dépenses, l'objectif d'évolution des dépenses de fonctionnement de 1,2\% pour les années 2018-2022 semble « atteignable ». La Cour craint même que la relative " aisance financière » des collectivités locales ne les incite pas à réduire leurs dépenses et leur endettement dans les prochaines années. Le dispositif d'encadrement contractuel, qui ne couvre que $41 \%$ des dépenses locales, devrait être amélioré et mieux tenir compte de l'intercommunalité et des situations individuelles de chaque collectivité. La Cour rappelle la nécessité d'une réforme des dotations donnant plus de place à la péréquation, d'une meilleure maîtrise de l'incidence financière des normes, d'une réforme profonde de la fiscalité locale, d'une plus grande fiabilité des comptes locaux (à laquelle contribueront la mise en œuvre des normes comptables, le 
compte unique et la certification), d'une plus grande cohérence entre les actions de l'État et celles des collectivités, comme le montre l'étude des missions des collectivités relatives à l'enseignement scolaire et aux activités périscolaires.

\section{$\lambda$ Les collectivités locales dans le projet de loi de finances pour 2019}

En 2019, les collectivités territoriales dégageront un excédent de capacité de financement de 0,1\% du PIB qui contribuera à la réduction du déficit public. Après le rebond de 2017, les dépenses de fonctionnement devraient ralentir à $+0,9 \%$. Les concours de l'État sont stabilisés conformément aux engagements pris : 48,19 Md€ (+100 M€); la DGF se maintient à $26,9 \mathrm{Md} €$; les dotations $d^{\prime}$ investissement au bloc communal (1,8 Md€) et aux départements $(0,3 \mathrm{Md} €)$ augmentent, de même que le fonds de compensation de la TVA (5,6 Md€, + $37 \mathrm{M} €)$. Le transfert de TVA aux régions est très dynamique (+ $166 \mathrm{M} €)$. La deuxième tranche de suppression de la taxe d'habitation (3,8 Md€) n'affectera pas les budgets locaux. La question de la compensation de la suppression pour les $20 \%$ de contribuables restants en 2020 sera prévue dans le projet de loi de réforme de la fiscalité locale qui sera présenté au $1^{\text {er }}$ trimestre 2019. Une simplification de la dotation d'intercommunalité est actée. L'automatisation du FCTVA est prévue pour 2020. Deux fonds sont créés pour accompagner la fermeture de certaines centrales énergétiques. La notion d'établissement industriel ou commercial pour la taxation des entrepôts aux impôts directs locaux est précisée.

\section{FINANCES SOCIALES}

\section{$\lambda$ Publication de la loi « pour la liberté de choisir son avenir professionnel $"$}

La Loi n²018-771 du 5 septembre 2018 pour la liberté de choisir son avenir professionnel, dite «Pénicaud 2 », a été promulguée sans les quelques cavaliers législatifs invalidés par le Conseil constitutionnel.

\section{$\lambda$ Les débats sur la réforme de l'Assurance chômage}

Dès le milieu de l'été, les débats ont démarré sur la réforme de l'Assurance chômage annoncée dans la loi «Pénicaud 2 ». (cf Repères d'août). Le 25 septembre, le ministère du Travail a adressé la lettre de cadrage qui doit guider la renégociation de la convention UNEDIC : il est prévu un montant d'économies de 3,9 Md€ sur 3 ans d'ici 2022. Quatre sujets devront, par priorité, être traités en profondeur : le fait que la possibilité de cumuler allocations de chômage et revenus d'activité courte combinée avec des droits rechargeables ne soit pas limitée dans le temps; le régime de l'activité "conservée » et les « droits rechargeables »; les règles de calcul du salaire journalier, dont les modalités actuelles facilitent excessivement les contrats courts; la question du montant et de la durée de l'indemnisation au regard du niveau de salaire antérieurement perçu. S'agissant des contrats courts, le document ne parle pas explicitement de «bonus/malus », mais les partenaires sociaux sont fermement invités à trouver un mécanisme, avec la menace implicite qu'à défaut, le gouvernement pourrait ne pas ratifier l'accord.

\section{$\lambda$ Les comptes de la santé pour 2017}

La DREES a publié le 10 septembre les comptes de la santé pour 2017. II en ressort que la consommation de soins et biens médicaux s'est élevée à $199 \mathrm{Md} €$, soit 8,7\% du PIB, avec une croissance plus faible que les années précédentes. Ce ralentissement est imputable à la décélération des dépenses hospitalières (+ 0,9\% par rapport à $+1,7 \%$ en 2016). La croissance des soins de ville $(+2,5 \%)$ restant très proche de celle des années précédentes. La Sécurité sociale prend globalement en charge $77,8 \%$ des dépenses de santé, les organismes complémentaires 13,2\%, l'État 1,5\%, ce qui fait que le reste à charge des ménages est de $7,5 \%$, en diminution constante depuis 2008 , du fait d'une proportion croissante de patients bénéficiant d'une exonération au titre des affections de longue durée. La France est l'un des pays de I'OCDE où le reste à charge est le plus faible, alors même que le poids des dépenses de santé est l'un des plus élevé.

\section{$\lambda$ Présentation du plan « Ma santé 2022 »}

Le 18 septembre, le Président de la République a rendu public le plan « ma santé 2022 ». Présentée comme la plus vaste réforme de la santé depuis 1958, le plan part du constat d'une organisation du système de soins désormais inadaptée aux défis que représentent le vieillissement de la population et la progression des pathologies de longue durée, l'inégalité croissante des territoires dans l'accès aux soins, le cloisonnement difficile à surmonter entre les différentes professions et une politique de maîtrise des dépenses qui repose excessivement sur l'hôpital. Le premier ensemble de mesures porte sur l'organisation et l'améliorations des soins de proximité : création d'ici 2022 de 1000 Communautés professionnelles territoriales de santé (CPTS) associant professionnels de ville et hospitaliers; institution d'une nouvelle fonction $d^{\prime}$ " assistants médicaux » pour 
libérer le temps des médecins; nouvelle répartition des missions entre les hôpitaux (soins de proximité, médecine, soins spécialisés, et plateaux techniques de pointe ultraspécialisés). Le second ensemble porte sur l'adaptation des métiers et des formations : suppression du numerus clausus et mise en place d'un recrutement beaucoup plus progressif, à partir de cursus diversifiés. La réforme du financement sera conduite progressivement à partir de la LFSS 2019 : la part de la tarification à l'activité (T2A) sera réduite, pour introduire un financement à la qualité (sur la base d'un nombre limité d'indicateurs) et un financement au forfait, mis en place dès l'an prochain pour certaines pathologies de longue durée. Le plan comporte également un important volet investissement hospitalier. Au total un montant de 3,4 Md€ seront mobilisés sur la période 20192022 (1,6 Md€ pour la structuration ville/hôpital dans les territoires, 0,5 Md€ pour la transformation numérique, 0,4 Md€ pour les métiers et les formations et $1 \mathrm{Md} €$ pour l'investissement). À cette fin, l'ONDAM sera relevé à 2,5\% en 2019.

\section{$\lambda$ Un rapport sur la réforme des aides sociales}

Le rapport commandé par le Premier ministre à M. Julien Damon, professeur associé à SciencesPo et Mme Christine Cloarec, députée d'Ille et Vilaine(LREM), a eu pour objet d'identifier des pistes pour pallier les dysfonctionnements de notre système d'aides sociales et de permettre à chacun de toucher « la juste prestation » (minima sociaux, prime d'activité, allocations familiales, allocation logement, allocation de retour à l'emploi); I'une de ses préconisations majeures porte sur la « contemporanéité » des versements, qui a pour objet de faire " coller» les montants versés au plus près des derniers revenus connus. C'est l'orientation qu' a adoptée la CNAF en prévoyant de prendre en compte, pour la liquidation des allocations logement, à partir du 1er avril 2019, les ressources des 12 derniers mois au lieu de celles de l'année N-2, ce qui devrait permettre de limiter les indus dus au changement de niveau de revenu (1,2 Md€ d'économies attendues).

\section{$\lambda$ Un rapport sur l'accompagnement des bénéficiaires du RSA}

Mme Claire Pitollat, députée des Bouches du Rhône (LREM) et M. Mathieu Klein, Président du conseil départemental de Meurthe et Moselle, ont remis, fin août, au Premier ministre un rapport sur l'accompagnement des bénéficiaires du RSA. Ce rapport comporte 50 propositions, portant notamment sur la mobilisation à l'accès aux droits, la priorité à donner à l'accompagnement avec pour finalité le retour à l'emploi et la gouvernance du dispositif, avec en perspective une recentralisation du financement de solidarité sur l'État.

\section{$\lambda$ Prestations sociales et réduction de la pauvreté}

Une étude de la DREES du 7 septembre évalue le rôle des prestations familiales et des minima sociaux pour la réduction de la pauvreté. En 2015, on comptait 8,9 millions de personnes $(14,2 \%$ de la population) vivant en dessous du seuil de pauvreté. L'étude de la DREES, qui comporte beaucoup d'informations sur les conditions de vie et d'emploi, ainsi que les trajectoires des bénéficiaires de minima sociaux, estiment que les prestations sociales et la fiscalité réduisent de 8,1 points le taux de pauvreté, avec un effet particulièrement sensible pour les familles monoparentales et les personnes de moins de 20 ans.

\section{$\lambda$ Le «plan pauvreté "}

Partant du constat que la France est marquée par la « reproduction de la pauvreté » qui touche un nombre important d'individus dès l'enfance et se perpétue de génération en génération, ainsi que par un système peu lisible de prestations et de minima sociaux, la «Stratégie nationale » présentée le 13 septembre comporte 5 engagements fondamentaux. Les deux premiers concernent la petite enfance (formation de 600000 professionnels de la petite enfance pour l'apprentissage du langage avant la maternelle, développement des modes de garde et du nombre de places en crèche, actions pour améliorer l'alimentation des enfants à l'école, mesures pour l'hébergement...). Le $3^{e}$ engagement vise à garantir un parcours de formation pour tous les jeunes, en instaurant une obligation de formation jusqu'à 18 ans, ainsi qu'en développant les mécanismes d'accompagnement (en particulier la "garantie jeunes ») pour les plus de 18 ans les plus vulnérables (" décrocheurs »). Le 4 e engagement - qui a fait l'objet $d^{\prime}$ intenses commentaires - porte sur la création d'un « revenu universel d'activité » d'ici 2022 : celui-ci reposera sur la refonte complète des minima sociaux (RSA, prime d'activité, aides au logement...) et il s'accompagnera de la revalorisation de la prime d'activité jusqu'à la fin du quinquennat ainsi que de l'intégration de l'aide au paiement d'une complémentaire santé (ACS) - jugée trop complexe - à la couverture maladie universelle complémentaire (CMU-C). Enfin le dernier engagement a pour objet d'accompagner, par une " garantie d'activité » tous les allocataires de minima sociaux vers l'emploi, le bénéficiaire du revenu universel d'activité ayant luimême l'obligation d'agir pour son insertion. Au total, avec les divers instruments dont la création 
est prévue (Fonds d'investissement social, Fonds de lutte contre la pauvreté et l'emploi...), la Stratégie nationale mobilisera 8,5 Md€ d'ici la fin du quinquennat (dont 3,9 $\mathrm{Md} €$ pour la seule revalorisation de la prime d'activité.

\section{$\lambda$ Le rapport Charpy-Dubertret sur les relations financières entre l'État et la sécurité sociale}

Le rapport sur les « relations financières entre l'État et la Sécurité sociale » remis au début septembre par MM. Christian Charpy et Julien Dubertret était prévu par l'article 27 de la loi de financement de la Sécurité sociale pour 2018. II s'agit d'organiser les transferts financiers de la Sécurité sociale vers l'État. En effet, les prévisions de la Loi de programmation des finances publiques de 2018 ont prévu un excédent croissant du solde des administrations de Sécurité sociale (ASSO) à partir de 2019, et le plafonnement de celui-ci à 0,8 points de PIB, le surplus devant être transféré à l'État, soit $3 \mathrm{Md} €$ en 2019, 14 Md€ en 2020, 20 Md€ en 2011 et plus de $27 \mathrm{Md} €$ en 2022. Les auteurs du rapport estiment qu'une partie importante des excédents ne pourra pas être transférée (ceux de I'UNEDIC et de l'AGIRC-ARRCO) et que d'autres apparaissent très aléatoires (surplus des hôpitaux par exemple). Le rapport suggère que les transferts s'opèrent par le biais de la compensation des exonérations et allègements de charge de l'État au profit de la SS, ceux-ci ne devant plus être compensés qu'à hauteur de $50 \%$ pour les nouveaux allègements au motif qu'ils « dopent » les créations d'emploi et donc augmentent les ressources de régimes sociaux. Le rapport propose ainsi de transférer, d'ici 2022, 12 Md€ d'excédents à l'État et d'amortir $15 \mathrm{Md} €$ de la dette sociale restant dans les comptes de l'ACOSS. Par ailleurs, les auteurs proposent une remise à plat du financement de la protection sociale, en renvoyant à l'État les impôts qui ont peu de rapport avec celle-ci (taxe sur les salaires...), et de compenser la perte de recettes ainsi générée par une affectation de TVA à hauteur de $35 \mathrm{Md} €$. Enfin le rapport propose, logiquement, d'élargir le périmètre des lois de financement de la SS à l'Assurance chômage et aux régimes complémentaire de vieillesse.

\section{$\lambda$ Le rapport de la Commission des comptes de la sécurité sociale de septembre 2018}

Le nouveau rapport de la Commission des comptes de la Sécurité sociale rendu public le 25 septembre reste dans la ligne de celui de juin dernier (cf Repères de juin), en confirmant le retour à une situation excédentaire (+ 2,4 Md€), cette année, du seul régime général. Pour l'exer- cice 2018, donc, par rapport aux prévisions de juin 2018, le solde global régime général + fonds de solidarité vieillesses ( $R G+F S V)$ varie très peu (- 0,4 Md€ contre - $0,3 \mathrm{Md} €$ en juin). Pour l'exercice 2019, les prévisions tendancielles retenues par la Commission des comptes de la SS - avant toute mesure nouvelle de la loi de financement de la SS - font apparaître un déficit prévisionnel de l'ensemble RG+FSV de - 3 Md€. Le RG repasserait dans le rouge avec un déficit de $-1,1 \mathrm{Md} €$, imputable à l'Assurance maladie (- 3,5 Md€ sur la base d'une croissance tendancielle de I'ONDAM de $+2,5 \%$ ) à l'Assurance vieillesse (- 0,3 Md€ mais avec l'hypothèse que les pensions seraient revalorisées comme l'inflation annuelle constatée à 1,5\%). En revanche, la branche Famille continuerait d'améliorer son redressement avec un excédent prévisionnel de 1,7 Md€ contre + 0,7 Md€ en 2018, de même que le FSV dont le déficit serait ramené à - 1,8 Md€), sous l'effet d'une amélioration de la situation de l'emploi et cela malgré l'augmentation prévue du minimum vieillesse. Les mesures nouvelles prévues dans le PLFSS 2019 ont largement corrigé cette prévision tendancielle (cf infra).

\section{$\lambda$ Le PLFSS pour 2019}

La ministre des Solidarités et de la Santé et le ministre des Comptes publics ont présenté, le 25 septembre, le PLFSS pour 2019, qui sera adopté par le Conseil des ministres au début octobre. Pour la première fois depuis 2001, I'ensemble régime général + fonds de solidarité vieillesse (RG+FSV) présenterait un solde excédentaire (+ 0,7 Md€). Le Régime général dégagerait un excédent de 2,5 Md€ (comparable à celui de 2018) : - 0,5 Md€ pour l'Assurance maladie, $+1,1 \mathrm{Md} €$ pour la branche AT-MP, + 0,7 Md€ pour la branche Vieillesse, + 1,2 Md€ pour la branche Famille. Le déficit du FSV serait ramené à - 1,8 Md€. Par ailleurs, les ministres ont confirmé l'objectif d'éteindre la dette sociale en 2024. À cet effet, la moitié de la dette résiduelle portée par I'ACOSS - soit $15 \mathrm{Md€} \mathrm{-} \mathrm{serait} \mathrm{transférée} \mathrm{à} \mathrm{la}$ CADES sur 3 ans. En outre, le PLFSS 2019 met en œuvre l'une des préconisations fondamentales du rapport Charpy-Dubertret (cf supra), à savoir la mise à la charge de la SS d'une partie des exonérations de charges sociales. Ainsi, en 2019 le financement des exonérations de cotisations sociales des heures supplémentaires et, en 2020, une partie des allègements de cotisations consécutifs à la transformation du CICE en allègements généraux seront mis à la charge du Régime général. Pour ce qui concerne l'Assurance maladie, on en resterait à une évolution modéré de I'ONDAM (+2,5\% en 2019), avec un meilleur équilibre entre I'hôpital et la médecine de ville et une légère progression par rapport à l'ONDAM de 2018 (+ 2,3\%) 
qui permettra de commencer à financer les mesures pour le renforcement des soins de proximité annoncées dans le plan santé, ainsi que des investissements des établissements dans l'immobilier et le numérique. La LFSS 2019 instaure par ailleurs des dispositifs de tarification au forfait pour réduire la part de la tarification à l'activité; de même, la mise en place du « reste à charge zéro » pour les frais dentaires, d'optique et d'audioprothèse commencera à se déployer en 2019 pour être complète en 2021. S'agissant de l'Assurance vieillesse, la limitation à $0,3 \%$ de la revalorisation des pensions va permettre d'économiser environ $3 \mathrm{Md} €$, tout en garantissant la poursuite de la revalorisation des minima sociaux - minimum vieillesse, $\mathrm{AAH}$...), ainsi que du revenu de solidarité active conformément aux engagements du Président de la République. Le PLFSS 2019 met en œuvre d'importantes mesures d'allègement des charges destinées à renforcer la compétitivité des entreprises et l'encouragement à l'activité. Annoncé l'an dernier, le basculement du CICE sur des allègements généraux de cotisations sociales s'opèrera en 2 temps, au $1^{\text {er }}$ janvier 2019 pour l'allègement de 6 points de cotisation d'assurance maladie dans la limite de 2,5 SMIC, et au $1^{\text {er }}$ octobre, pour les allègements généraux de cotisations sociales au niveau du SMIC. Par ailleurs, comme annoncé, la part salariale des cotisations d'assurance vieillesse et de base sur les heures supplémentaires sera exonérée à partir du $1^{\text {er }}$ septembre 2019, ce qui devrait représenter un gain de pouvoir d'achat de $2 \mathrm{Md} €$ en année pleine.

\section{FISCALITÉ ET PROCÉDURES FISCALES}

\section{$\lambda$ Poursuite de la baisse des impôts dans les pays de l'OCDE}

Le rapport Tax policy reform de l'OCDE qui couvre 38 pays de l'OCDE confirme la tendance à la baisse de la pression fiscale, notamment sur les entreprises : le taux moyen de l'impôt sur les sociétés dans les pays de l'OCDE est passé de $32,5 \%$ en 2000 à $23,9 \%$ en 2018. II s'agit plus d'une « course à la moyenne » qu'une course au «moins disant fiscal ». Les impôts sur le revenu ont en général baissé sur les revenus d'activité moyens ou faibles. L'OCDE déplore que la fiscalité environnementale reste concentrée sur l'énergie et les véhicules.

\section{$\lambda$ Feu vert pour le prélèvement à la source dans de bonnes conditions techniques?}

Un communiqué de la DGFiP du 2 septembre 2018 précise que « contrairement à ce qui est relayé dans la presse, il est impossible que ces doublons conduisent à un double prélèvement des contribuables». Ce même communiqué précise que " c'est en raison du bilan positif fait à l'issue des tests qu'a été confirmée la faisabilité de la mise en œuvre du PAS en janvier 2019 ». Le PAS a fait I'objet d'un ultime arbitrage entre le Président de la République et les ministres concernés. Le 4 septembre 2018, le Premier ministre a confirmé que ce nouveau mode de prélèvement serait bien mis en place le $1^{\text {er }}$ janvier 2019. Une étude de I'OFCE (Gilles Le Garrec, Vincent Touzé, « Le PAS de I'IR : peu d'avantages et beaucoup d'inconvénients ", OFCE, $n^{\circ} 24$, sept. 2018) établit un bilan des avantages et des inconvénients de la réforme. Elle montre que les avantages (une meilleure synchronisation entre impôt et revenu, une perception plus rapide des recettes d'IR pour l'État, une meilleure observation des revenus en temps réel) semblent peu nombreux au regard des inconvénients engendrés (tâches administratives supplémentaires pour les agents publics et privés; risque d'erreurs pouvant être sources de contentieux; année blanche due à la non-fiscalisation des revenus 2018; hausse fiscale liée à la suppression du délai d'un an pour payer l'IR; impact psychologique potentiel de l'effet fiche de paie avec un salaire versé réduit; divulgation d'information fiscale à l'employeur; non-mensualisation des remboursements de crédits d'impôt; nouvelles formes de complexité qui peuvent réduire le consentement des contribuables français à payer l'impôt). Selon le président de la commission des finances de l'Assemblée nationale, lors d'un entretien radiophonique du 7 septembre 2018, le maintien en l'état du système actuel de prélèvement, assorti d'un étalement automatique et plus facile du paiement de l'impôt en cas de forte baisse du revenu et une mensualisation généralisée sur 12 mois au lieu de 10, aurait eu le mérite de la simplicité.

\section{$\lambda$ Une large concertation sur la relation de confiance}

Le ministère de l'Action et des Comptes publics et la DGFiP ont lancé une consultation en ligne auprès des entreprises en vue de construire la fameuse « relation de confiance» souhaitée entre elles et l'administration fiscale. Ce dispositif, prévu par l'article 17 de la loi n²018-727 du 10 août 2018 pour un État au service d'une société de confiance dite "ESSOC », instaurant le « droit à l'erreur» en matière fiscale, habilite le Gouvernement à prendre par ordonnances toute disposition relevant du domaine de la loi en vue de renforcer la sécurité juridique des entreprises soumises à des impôts commerciaux. Depuis le 25 juillet 2018 et jusqu'au 31 octobre inclus, les TPE, PME, ETI et grands groupes sont invités à 
répondre à un questionnaire en ligne pour faire part de leurs besoins sur le plan fiscal. Au 18 septembre 2018, environ 250 entreprises avaient répondu. Le ministre a lancé le 12 septembre 2018 les travaux du groupe d'experts dans le cadre de la concertation publique pour la mise en place d'une nouvelle relation de confiance entre les entreprises et l'administration fiscale.

\section{$\lambda$ Une contribution à l'audiovisuel public sans téléviseur?}

Mi-septembre, la ministre de la Culture, Françoise Nyssen, a annoncé vouloir réformer la contribution à l'audiovisuel public (CAP), pour qu'elle soit " déconnectée de la détention d'un téléviseur ». Matignon, l'Élysée et Bercy ont indiqué que cette réforme n'est pas d'actualité. Annoncé sous le quinquennat précédent en 2014, puis finalement abandonné, ce projet de réforme concernerait les détenteurs d'ordinateurs, de tablettes et de téléphones mobiles. Chaque année, lors de l'examen de la loi de finances, Bercy et la Rue de Valois reculent prudemment et se contentent d'ajouter 1 à 2 euros au montant de la CAP, fixée pour 2018, à 139 euros. Le produit de la CAP en 2017 a été, selon la Cour des comptes, de 3,17 Md€ $(27,6 \mathrm{M}$ de foyers assujettis, dont 23,1 $\mathrm{M}$ de foyers payants, soit 4,4 M de foyers dégrevés sur rôle).

\section{$\lambda$ Proposition de modification des droits de succession}

Le secrétaire général du Mouvement «En marche », Christophe Castaner, a déclaré au mois de septembre 2018 vouloir lancer une réflexion sur la fiscalité des successions afin de lutter contre la "progression des inégalités de naissance ». Selon lui, l'imposition sur les successions " est complexe, elle est mal acceptée, elle n'a pas vraiment suivi l'évolution de la société et les nouvelles formes familiales», précisant qu'il ne s'agissait pas d'annonces du Gouvernement mais d'une piste de réflexion du parti majoritaire. Dans un communiqué, la présidence de la République a clarifié la position du Chef de l'État, en assurant que ce dernier « a formellement exclu toute modification des droits de succession sous sa présidence» ...

\section{$\lambda$ L'impact des réformes de l'imposition du capital}

Une étude de l'Observatoire économique de la Fondation Jean-Jaurès intitulée « Réforme de la fiscalité du capital : la sécession des riches » a été publiée le 20 septembre 2018. Selon celle-ci, les mesures fiscales d'allègement de la fiscalité de la détention et des revenus du capital entrées en vigueur le $1^{\text {er }}$ janvier 2018 vont coûter 4,5 Md€ par an au budget de l'État. Ces mesures profitent principalement à moins de 400.000 ménages parmi les plus riches. Selon la Fondation JeanJaurès, cette réforme manque sa cible - stimuler I'investissement - et va provoquer un inévitable accroissement des inégalités de revenu et de patrimoine.

\section{$\pi$ Vers un Observatoire de la fraude fiscale}

Le 13 septembre 2018, lors d'un discours, le ministre de l'Action et des Comptes publics, a annoncé le lancement d'un Observatoire dont le but sera de «produire une évaluation publique de la fraude fiscale et sociale ». Le syndicat Solidaires Finances Publiques s'est félicité d'une telle annonce. Ce dernier réclame une initiative de ce type depuis la fin des années 1990 (cf. son rapport de janvier 2013 et de septembre 2018) estimant le manque à gagner entre 60 et $80 \mathrm{Md} €$ chaque année. Selon lui, l'Observatoire de la fraude fiscale doit déterminer un cadre précis de travail car, selon le périmètre retenu et la définition plus ou moins restrictive de la fraude, le montant du manque à gagner qui en découlera sera plus ou moins important. .

\section{$\lambda$ Les principales mesures fiscales du PLF pour 2019}

Le projet de loi de finances pour 2019 présenté au conseil des ministres du 24 septembre 2018 contient des mesures fiscales moins significatives que le précédent. En premier lieu, s'agissant de la fiscalité des particuliers, outre des ajustements au barème de l'impôt sur le revenu, le PLF accompagne la mise en place du prélèvement à la source (PAS) : acompte de $60 \%$ pour certains avantages fiscaux dès le 15 janvier et décalage d'un an du PAS pour les particuliers employeurs (art. 3 PLF 2019). II vise à mieux cibler l'action de l'État en faveur du soutien à l'économie ultramarine et aux habitants de ces territoires, en excluant les plus hauts revenus (art. 4 PLF 2019). Par ailleurs, l'imposition des plus-values sur valeurs mobilières et droits sociaux en cas de transfert du domicile fiscal hors de France, dite " exit tax», sera remplacée par un nouveau dispositif, dit antiabus, d'imposition des plus-values latentes sur les titres et valeurs mobilières, limité aux résidents français qui cèdent leurs titres moins de deux ans après leur départ (art. 51 PLF 2019). En deuxième lieu, s'agissant de la fiscalité des entreprises, le PLF 2019 contient le volet fiscal du projet de loi relatif à la croissance et la transformation des entreprises, dit «PACTE » et plusieurs dispositions de mise en conformité avec le droit européen : fiscalité des groupes (art. 12 PLF 2019) ; plafonnement de la déductibilité des charges financières à $30 \%$ du résultat ou à 3 millions d'euros si 
ce montant est supérieur (art. 13 PLF 2019) ; aménagement du régime $d^{\prime}$ imposition des produits tirés de l'exploitation et de la cession de brevets qui sera proportionné aux dépenses de recherche et de développement réalisées sur le territoire national (art. 14 PLF 2019). L'impôt sur les sociétés sera réformé et sécurisé, dans une logique de convergence européenne (art. 15 PLF 2019). Le «pacte Dutreil», qui assure la pérennité d'une activité sous le contrôle d'un noyau dur d'actionnaires, est aménagé : maintien d'une exonération partielle des droits de mutation en cas de cession entre héritiers ou donataires, assouplissement des modalités d'apport à une holding en cours d'engagement, suppression de l'obligation de déclaration administrative annuelle (art. 16 PLF 2019). Les entreprises qui optent pour I'IS pourront, durant cinq ans, revenir à I'IR, si ce choix se révèle pénalisant a posteriori (art. 17 PLF 2019). L'exonération de TVA sur les services à la personne sera réservée aux seules prestations rendues à des publics fragiles par des associations bénéficiant d'une autorisation ou d'un agrément (art. 20 PLF 2019). Les aides fiscales en faveur de la gestion des risques et de l'investissement agricole seront réformées, afin d'inciter les exploitants à constituer une épargne pour faire face aux crises ou difficultés éventuelles (art. 18 PLF 2019). Par ailleurs, un plafonnement de l'abattement sur le bénéfice des jeunes agriculteurs est prévu (art. 53 PLF 2019). Le crédit d'impôt pour le rachat d'une entreprise par leurs salariés sera étendu : la condition tenant à la reprise par un nombre minimum de 15 salariés sera supprimée (art. 49 PLF 2019). En troisième lieu, s'agissant de la fiscalité environnementale, à destination des particuliers, le Gouvernement prévoit le renforcement du barème du malus automobile, la prorogation d'un an du crédit d'impôt pour la transition énergétique (CITE) sans changement de périmètre, avant sa conversion en prime pour les ménages modestes (art. 57 PLF 2019), la prorogation de l'éco-prêt à taux zéro (PTZ) qui sera réservé aux logements de plus de deux ans, sans condition de bouquet de travaux au $1^{\text {er }}$ mars 2019 (art. 58 PLF 2019), la suppression des tarifs réduits de taxe intérieure de consommation sur les produits énergétiques (TICPE) dont bénéficient certains carburants polluants utilisés pour la propulsion des véhicules non routiers, sauf pour les entreprises des secteurs ferroviaire et agricole (article 19). Enfin, s'agissant de la fiscalité environnementale à destination des entreprises, le PLF prévoit l'augmentation des tarifs de la composante « déchets » de la taxe générale sur les activités polluantes (TGAP-d) entre 2021 et 2025 pour inciter à la valorisation plutôt qu'à l'incinération et au stockage des déchets (art. 8 PLF 2019), le relèvement des tarifs de la composante de la
TGAP visant à encourager le recours aux biocarburants, renommée " taxe incitative à l'incorporation de biocarburants » (art. 60 PLF 2019) et la réduction à 5,5\% du taux de TVA sur certaines prestations de gestion des déchets (art. 59 PLF 2019). En dernier lieu, au titre des mesures de simplification fiscale, actées dans le cadre du programme « Action Publique 2022 », le PLF 2019 entend moderniser le recouvrement de l'impôt, en réduisant le nombre d'interlocuteurs pour le redevable, en développant les téléprocédures et en faisant en sorte que la DGFiP n'ait plus à manier d'espèces d'ici deux à trois ans. Le Gouvernement prévoit la suppression de 20 taxes à faible rendement (art. 9 du PLF) et la suppression de niches fiscales inefficientes (remise gratuite par l'employeur de matériels informatiques amortis; certaines aides à l'installation de salarié; certains avantages liés aux sociétés financières d'innovation (SFI) et aux sociétés coopératives d'intérêt collectif (SCIC); des dispositifs relatifs à l'acquisition et à la construction de logements sociaux dans les départements d'outre-mer) (art. 11 PLF 2019). Enfin, le Gouvernement prévoit le transfert du recouvrement des diverses contributions sur les boissons non alcooliques des services de la DGDDI vers les services de la DGFiP (art. 62 PLF 2019).

\section{$\lambda$ Rapport parlementaire sur l'évasion fiscale internationale des entreprises}

Le Rapport d'information n 1236 de Mme Bénédicte Peyrol et M. Jean-François Parigi, députés, déposé le 12 septembre 2018 et relatif à l'évasion fiscale internationale des entreprises s'efforce de préciser la notion d'évasion fiscale, «toutes les opérations qui, bien que légales, sont artificielles et sans substance et doivent ainsi être fiscalement sanctionnées » et formule quinze propositions destinées à renforcer les moyens de la combattre: contrecarrer la manipulation des prix de transfert, assouplir la notion d'abus de droit (« motivation fiscale principale et non plus exclusive »), renforcer l'attractivité de la France (maintien d'un régime favorable aux brevets en y incluant les logiciels), soutenir les projets européens ACCIS et de taxation des services numériques; renforcer les sanctions contre les paradis fiscaux; accroître la transparence (registres des bénéficiaires effectifs, déclaration de montages fiscaux à risque, publicité des déclarations pays par pays).

\section{Management public}

\section{$\lambda$ Revue de missions à Bercy}

Le 4 septembre 2018, dans un discours devant les organisations syndicales, le ministre de l'Écono- 
mie et des finances, Bruno Lemaire, a donné des indications sur les suites de la circulaire du Premier ministre du 24 juillet 2018 (voir Repères de juillet 2018) et du rapport Action publique 2022. Les missions économiques des DIRECCTE seraient allégées pour devenir complémentaires de celles des régions et circonscrites à l'accompagnement des entreprises en difficulté, au développement des filières stratégiques et à l'innovation. Les agents touchés par la réduction des emplois (de 430 à 120) bénéficieront d'un plan d'accompagnement social. Les attributions de la Direction générale de la concurrence, de la consommation et de la répression des fraudes seront recentrées sur les missions prioritaires qui restent à définir. Un certain nombre de contrôles seront confiés à d'autres acteurs. Les implantations du réseau économique à l'étranger seront réexaminées et les fonctions supports seront transférées aux ambassadeurs, ce qui permettrait d'économiser 75 équivalents temps pleins.

\section{$\lambda$ Poursuites des simplifications administratives}

Le Conseil des ministres du 12 septembre 2018 a adopté deux mesures qui s'inscrivent dans les efforts patients et continus de simplification administrative. Un projet de loi de ratification de l'ordonnance $n^{\circ}$ 2018-474 du 12 juin 2018 harmonise à droit constant les définitions des revenus utilisées pour l'assiette des cotisations de sécurité sociale, de la contribution sociale généralisée et de la contribution à la réduction de la dette sociale. Un projet de décret (D. $n^{\circ}$ 2018-785 du 12 septembre 2018, JORF du 13 septembre 2018, texte $\mathrm{n}^{\circ} 1$ ) supprime 20 commissions consultatives. Depuis 2012, le nombre de commissions consultatives serait passé de 670 mi-2012 à 389. Le Premier ministre a complété sa circulaire du 24 octobre 2017 par une nouvelle circulaire du 12 septembre 2018 qui étend aux commissions consultatives le principe " one in, two out »(sic) qui s'applique depuis septembre 2017 aux textes règlementaires.

\section{$\lambda$ Nouveau concours de l'ENA réservé aux docteurs}

Un décret n²018-793 du 14 septembre 2018, JORF du 16 septembre 2018, texte $n^{\circ} 1$, prévoit l'organisation d'une nouvelle voie d'accès à l'École nationale d'administration qui s'ajoute aux trois existantes (externe; interne; expérience professionnelle, associative, syndicale ou élective). Elle sera réservée aux titulaires d'un doctorat et se déclinera en trois spécialités : sciences de la matière et de l'ingénieur; sciences de la vie; sciences humaines et sociales.

\section{$\lambda$ Le programme du Gouvernement pour la fonction publique}

Dans plusieurs déclarations, le Secrétaire d'État chargé de la fonction publique, Olivier Dussopt, a présenté le programme du Gouvernement qui concerne les trois versants de la fonction publique et qui fera l'objet d'un projet de loi sur la transformation de la fonction publique au printemps 2019. II s'appuiera sur « quatre leviers majeurs de refondation du contrat social avec les agents publics» : un dialogue social simplifié et recentré, un recours élargi au contrat, une rémunération plus individualisée, un accompagnement renforcé en matière d'évolution professionnelle. Une quarantaine de réunions ont eu lieu avec les organisations syndicales et les employeurs publics. La concertation se poursuivra après les élections professionnelles du 6 décembre 2018. Ces projets devront s'articuler avec quatre dossiers transversaux interministériels : simplification et amélioration de la qualité du service rendu, transformation numérique, organisation territoriale des services publics, modernisation de la gestion budgétaire et comptable et avec les feuilles de route ministérielles.

\section{$\lambda$ Pour un numérique inclusif}

De plus en plus de formalités administratives sont obligatoirement dématérialisées alors qu'on estime que 13 millions de Français n'utilisent pas ou peu internet et sont victimes "d'illectronisme ». Le Défenseur des droits, pour qui 33\% des Français de sont pas à l'aise avec internet, recommande l'instauration obligatoire d'une voie alternative aux services numériques dans une décision $n^{\circ} 2018-226$ du 3 septembre 2018 relative à la dématérialisation des formalités administratives. Le gouvernement a présenté le 13 septembre 2018 un plan national pour un numérique inclusif. Il repose sur l'initiative privée qui sera mobilisée via des appels à projet pour détecter, conseiller et outiller les acteurs de terrain et créer des ateliers numériques (projet « Hub France connectée »). Des actions de formation seront organisées et pourront être financées par un " pass numérique ». Les intermédiaires qui accompliront des démarches pour le compte d'autrui pourront bénéficier d'une connexion sécurisée par «France connect aidants ».

\section{EUROPE}

\section{入 Budget 2019 : le Conseil adopte sa position}

Lors d'une réunion à Bruxelles tenue le 4 septembre 2018, le Conseil ECOFIN a adopté sa position 
qui s'élève à un total de 164,1 milliards d'euros (Md€) de crédits d'engagement et 148,2 Md€ de crédits de paiement. Cela représente une augmentation respectivement de 2,09\% et 2,34\% par rapport à 2018. Si certains domaines bénéficieraient ainsi d'un soutien accru (par exemple recherche et l'innovation, $79 \%$, le programme Erasmus, $10,37 \%$, les investissements dans l'infrastructure, $26,46 \%$, I'environnement et l'action climatique, $5,20 \%$, ainsi que la gestion des migrations, 55,80\%), par rapport au projet de budget 2019 présenté par la Commission, le total des crédits d'engagement a tout de même été réduit de 1,6 Md€ et ceux de paiement de 0,5 Md€. Le Parlement - dont plusieurs membres ont critiqué les coupes opérées par le Conseil - devrait adopter ses amendements le 24 octobre. Cela devrait déclencher une période de conciliation de trois semaines, qui commencera le 30 octobre et prendra fin le 19 novembre.

\section{$\lambda$ Une note du CAE sur une nouvelle règle d'or européenne}

Le Conseil d'analyse économique (CAE) a présenté le 10 septembre 2018 une note au Premier ministre formulant des propositions pour réformer le budget européen autour des principes de simplification, de stabilisation et de soutenabilité. Selon le CAE, les règles rigides ont engendré une austérité budgétaire excessive pendant la crise et la réduction de la dette a été insuffisante dans de nombreux pays en période de conjoncture favorable dans les années 2000. Ces règles souffrent en outre de "gros problèmes de mesure », en étant basées sur le solde public structurel, «qui n'est pas observable et fait l'objet d'importantes erreurs d'estimation ». Étant ainsi « extrêmement complexe, peu transparent et conduisant à des erreurs de politique économique », ce cadre budgétaire européen devrait, selon les auteurs de la note, être largement réformé sur la base d'une " profonde simplification avec l'adoption d'une règle plus lisible et pilotable, centrée sur l'évolution des dépenses publiques». En pratique, il s'agirait de fixer des objectifs par pays en fonction de la situation économique observée. Ainsi chaque gouvernement proposerait un objectif à moyen terme de réduction du ratio dette/PIB qui permettrait de mieux adapter l'encadrement européen des politiques budgétaires.

\section{入 Aides d'État : le Luxembourg n'a pas accordé un traitement fiscal sélectif à McDonald's}

Le 19 septembre 2018, la Commission a conclu que l'absence d'imposition de certains bénéfices de McDonald's au Luxembourg n'a pas entraîné d'aide d'État illégale, étant donné qu'elle était conforme à la législation fiscale nationale et à la convention sur les doubles impositions entre le Luxembourg et les États-Unis. La Commission s'est même félicitée à cette occasion des mesures prises par le Grand Duché pour éviter à l'avenir toute double non-imposition.

\section{$\pi$ Aides d'État : aide incompatible de I'Italie à l'autorité portuaire de Naples}

Le 20 septembre, la Commission européenne a constaté que 44 millions d'euros de subventions octroyés au port de Naples, en vue de rénover les cales sèches louées aux chantiers navals Cantieri del Mediterraneo, étaient contraires aux règles de l'UE en matière d'aides d'État. Mais, en revanche, elle a considéré que le report d'encaissement des redevances de concession par l'autorité portuaire ne constituait pas une aide d'État.

\section{$\lambda$ Fraude à la TVA : 150 milliards de pertes}

Dans un rapport présenté le 21 septembre 2018, la Commission a incité les États membres à adopter la réforme qu'elle leur avait proposée l'an dernier (ce nouveau système définitif de TVA impliquant de taxer les ventes de biens à partir d'un pays de l'Union vers un autre de la même manière que si les biens étaient vendus au sein d'un seul et même État membre) en raison de leurs pertes de recettes de TVA que ses services ont évalués à près de $150 \mathrm{Md} €$ en 2016 . Cette perte appelée " écart de TVA » correspond à la différence entre les recettes de TVA attendues et le montant effectivement perçu.

\section{$\lambda$ Le projet de budget de l'Italie}

Le 7 septembre les gouvernements européens déclaraient avoir confiance en Rome pour respecter les règles budgétaires de l'Union, notamment parce que le ministre des Finances G. Tria annonçait un déficit ne devant pas dépasser 1,6\% du PIB. Mais les déclarations ultérieures du gouvernement italien, le 30 septembre (annonce d'un déficit égal à $2,4 \%$ du PIB les trois prochaines années sur la base d'une croissance de 1,6\% en 2019 et 1,7\% l'année suivante, bien supérieurs aux dernières prévisions revues à la baisse de $+1 \%$ et + 1,1\% en 2019 et 2020), ont amené les ministres des Finances de la zone euro à lui demander le 2 octobre de revoir son budget (voir Repères d'octobre). 


\section{INTERNATIONAL}

\section{$\lambda$ Crise monétaire en Argentine}

Alors que le peso argentin a perdu $50 \%$ de sa valeur et que l'inflation dépasse les 30\%, l'Argentine est en récession : le gouvernement prévoit un PIB réduit de $1 \%$ en 2018. Le président Mauricio Macri a donc annoncé le 3 septembre un vaste plan d'austérité afin de stabiliser l'économie et de lutter contre le déficit budgétaire. II comprend rien moins que la suppression d'une douzaine de ministères (12 sur 22), accompagnée d'une réduction des investissements (grands chantiers reportés) et des subventions de l'État (spécialement en matière de gaz et d'électricité), ainsi que de l'augmentation des taxes à l'exportation (retour d'une taxation à la source). Pour limiter les effets de ces mesures, l'augmentation des allocations et des programmes alimentaires, ainsi que le plafonnement du prix de certains produits de base ont été annoncés. Le ministre de l'Économie a fixé comme objectif l'équilibre budgétaire dès 2019, alors que l'accord avec le FMI conclu en juin pré- voyait de le réduire à $2,7 \%$ du PIB cette année, puis à 1,3\% en 2019, et de parvenir à l'équilibre en 2020. Avec un déficit passé de 6\% en 2015 à $3,9 \%$ en 2017 , ce nouvel objectif semble à court terme très difficile à atteindre.

\author{
Michel Le Clainche \\ avec le concours de \\ Aurélien Baudu (fiscalité), \\ Fabrice Bin (Europe et international), \\ Jean-François Calmette (finances de l'État) \\ et Yves Terrasse (finances sociales)
}

Retrouvez la chronique "Repères d'actualité " chaque mois dès le $15 \mathrm{du}$ mois suivant et toutes les chroniques depuis janvier 2015 avec des liens hyper textes aux documents cités sur le site www.gestionfinancespubliques.info

\section{$>$ Le Prix de thèse de la Cour des comptes}

Afin de contribuer à I'amélioration des connaissances et à I'innovation en matière de finances publiques ou de gestion publique, la Cour des comptes soutient la recherche dans ces domaines. Après le succès de la première session du prix de thèse de la Cour en 2017, un nouveau prix de thèse sera remis par son Premier président en 2019.

Ce prix est destiné à récompenser les auteurs de thèse, quelle que soit leur filière académique, qui contribuent à la meilleure compréhension de la gestion et des finances publiques, au renouvellement de l'approche théorique dans ces domaines et au développement de propositions opérationnelles.

La langue de publication de la thèse est le français. Sous réserve qu'un résumé long en français y soit adjoint, que la thèse ait été soutenue devant une université francophone et qu'elle ait donné lieu à au moins une publication en langue française, une thèse soutenue dans une autre langue pourra être examinée par le jury.

Le prix, d'un montant de $5000 €$, et, le cas échéant, un prix spécial d'un montant de $2000 €$, seront remis sur la base des délibérations d'un jury associant magistrats et universitaires de haut niveau.

Les docteurs souhaitant concourir doivent soumettre leur thèse pour le 31 mars 2019. La remise des prix aura lieu en juin 2019, au palais Cambon.

Le règlement du prix, les modalités et le calendrier précis de l'appel à projet sont présentés sur le site internet des juridictions financières www.ccomptes.fr, rubrique «nos activités». 